\title{
Détermination des paramètres météo nécessaires à la prévision du risque d'avalanche à échéance d'un jour
}

\author{
par Yves Durand et Laurent Mérindol \\ MÉTÉO-FRANCE :CNRM/CEN
}

\section{INTRODUCTION}

L'évolution du manteau neigeux dépend principalement des conditions météorologiques qui vont influencer dans chaque couche le bilan énergétique et massique, la stratigraphie et les caractéristiques physiques ainsi que la teneur en eau liquide. Toutes ces quantités sont importantes pour la prévision des risques d'avalanches hors des domaines sécurisés (PRA) qui est une des missions de Météo-France (MF). Comme pour la prévision météorologique, les modèles numériques de simulation de la neige sont depuis plusieurs années des outils performants et relativement fiables qu'utilisent les prévisionnistes des stations de montagne de MF en charge de ce risque particulier. L'équipement progressif des stations en moyens informatiques de pointe va leur permettre d'utiliser pleinement les possibilités de ces programmes dont les résultats complètent leur propre expertise notamment dans les cas de zones à faible densité d'observation ou en dehors de la saison hivernale.

\section{I 口 CHAÎNE ACTUELLE D'«ANALYSE »}

Le système est composé de trois modules distincts qui simulent les principales caractéristiques du couvert neigeux à l'échelle du massif $\left(\sim 400 \mathrm{~km}^{2}\right.$, cf fig 4$)$ et de ses principales pentes et expositions. Cette échelle de travail est compatible avec la finesse de notre estimation initiale des paramètres météorologiques. Elle permet d'appréhender les principales structures du manteau à l'exception de celles forcées par des effets locaux : transport de neige par le vent ou différents effets orographiques et géographiques.

The avalanche hazard estimation for the present and the coming days is one of the main tasks of the avalanche forecaster. For four years, some of them, in the alpine massifs of France, have been using a serie of automatic numerical models. These programs describe in real time the main meteorological conditions (Safran), the evolution of the snow cover (Crocus) and the resulting avalanche risks (Mepra) at different elevations, slopes and aspects of the considered massifs.

This paper presents the latest evolution of this automatic tool. This new version now makes it possible to provide one-day forecast states of snow cover over both the Alps and Pyrenees massifs including the main characteristics of the snowpack and an assessment of the corresponding avalanche hazards.

To achieve this result, the main changes concerned Safran. Two combined methods are used inside the same package : adaptations of larger scale meteorological forecasts and use of observations of analogous weather situations of the past. These two approaches are complementary especially for the evaluation of precipitation where the second solution has important fine scale information while the first exhibits important local biases. Validation of this new application was carefully done and proved the quality of the method, now used in real time by local forecasters. 
L'évolution du manteau neigeux est simulée par le modèle Crocus $[1,2]$ en fonction des conditions météorologiques qui lui sont fournies au pas horaire. Ces paramètres sont : la température de l'air ainsi que le vent (direction et force) et l'humidité, les précipitations (intensité et phase), la nébulosité intégrée, le rayonnement solaire descendant (direct et diffus) ainsi que le rayonnement infrarouge atmosphérique descendant. En plus des échanges d'énergie et de masse sur ses interfaces, le modèle simule différents processus physiques internes au couvert neigeux comme la conduction thermique, le tassement, la quantité d'eau sous forme liquide et sa percolation, la fonte et les effets dus au regel. La stratigraphie et les métamorphoses sont également modélisées ce qui améliore beaucoup les calculs d'albédo et de tassement et facilite l'interprétation des sorties. Cette estimation du type et de la taille des grains est une des grandes originalités de ce modèle.

Le diagnostic d'avalanche est établi par le modèle expert Mépra [3] qui examine la stabilité des différentes couches simulées par Crocus avec leurs caractéristiques physiques et morphologiques. Un diagnostic de risque naturel et accidentel est ainsi porté sur chaque profil du manteau neigeux avec une justification du raisonnement.

Les conditions météorologiques nécessaires à Crocus sont estimées (= analysées) par le modèle Safran [4] qui utilise un maximum d'informations observées (par différents réseaux d'observation) et prévue par le modèle Arpège [5] de prévision numérique du temps de MF. La démarche de Safran peut être vue comme un processus à deux étapes : dans un premier temps on calcule avec Arpège une ébauche des quantités que l'on veut analyser au moyen de différents opérateurs d'interpolation, puis ensuite on modifie cette première ébauche avec les observations disponibles. Dans le cas des précipitations, la première étape est remplacée par l'utilisation d'une climatologie spécifique par type de temps jugée préférable au modèle Arpège.

Les trois modèles Safran/Crocus/Mépra (chaîne SCM) sont activés quotidiennement, ce qui permet de suivre «au jour le jour " l'état du manteau neigeux dans 23 massifs alpins et 12 pyrénéens (cf. fig. 4) qu'il peut ainsi modéliser quelques heures après que les observations soient effectuées et transmises.

\section{II — NÉCESSITÉ D'UNE CHAÎNE DE PRÉ- VISION}

Il s'est bientôt avéré que cette connaissance de l'état passé de quelques heures du manteau neigeux était insuffisante surtout lorsque la situation évoluait rapidement et qu'il fallait donc envisager un mode "prévision " afin de couvrir la journée à venir et ainsi de fournir des éléments en temps réel au prévisionniste nivo. Le système à développer doit assurer une parfaite continuité temporelle avec sa version "analyse " et doit également être cohérent avec la prévision météo de plus grande échelle, ce qui exclut toute solution locale totalement déconnectée des modèles numériques de MF. Pour résoudre ce problème nous avons donc identifié deux sources principales d'information :

- le modèle Arpège, déjà mentionné, dont la résolution d'environ $30 \mathrm{~km}$ est insuffisante pour le travail à notre échelle ; son relief interne ne peut pas par exemple représenter les grandes vallées alpines. Il est cependant capable de bien appréhender et simuler les perturbations synoptiques qui vont affecter les chaînes alpines ou pyrénéennes et de donner une bonne représentation des variables météo en altitude. Il peut donc apporter un ensemble de valeurs moyennes, à charge pour nous de les adapter à échelle plus fine.

- les situations analogues du passé. La méthode n'est pas nouvelle et a déjà été essayée avec succès $[6,7]$; elle consiste à rechercher dans le passé une situation qui «ressemble " à celle de la journée en cours depuis 6 "heures " UTC jusqu'au lendemain à 6 UTC. On y arrive en minimisant une distance entre des journées du passé pour lesquelles on a des observations et une situation présente. Cette recherche se fait généralement sur une zone géographique assez grande afin de "retrouver " des phénomènes synoptiques similaires.

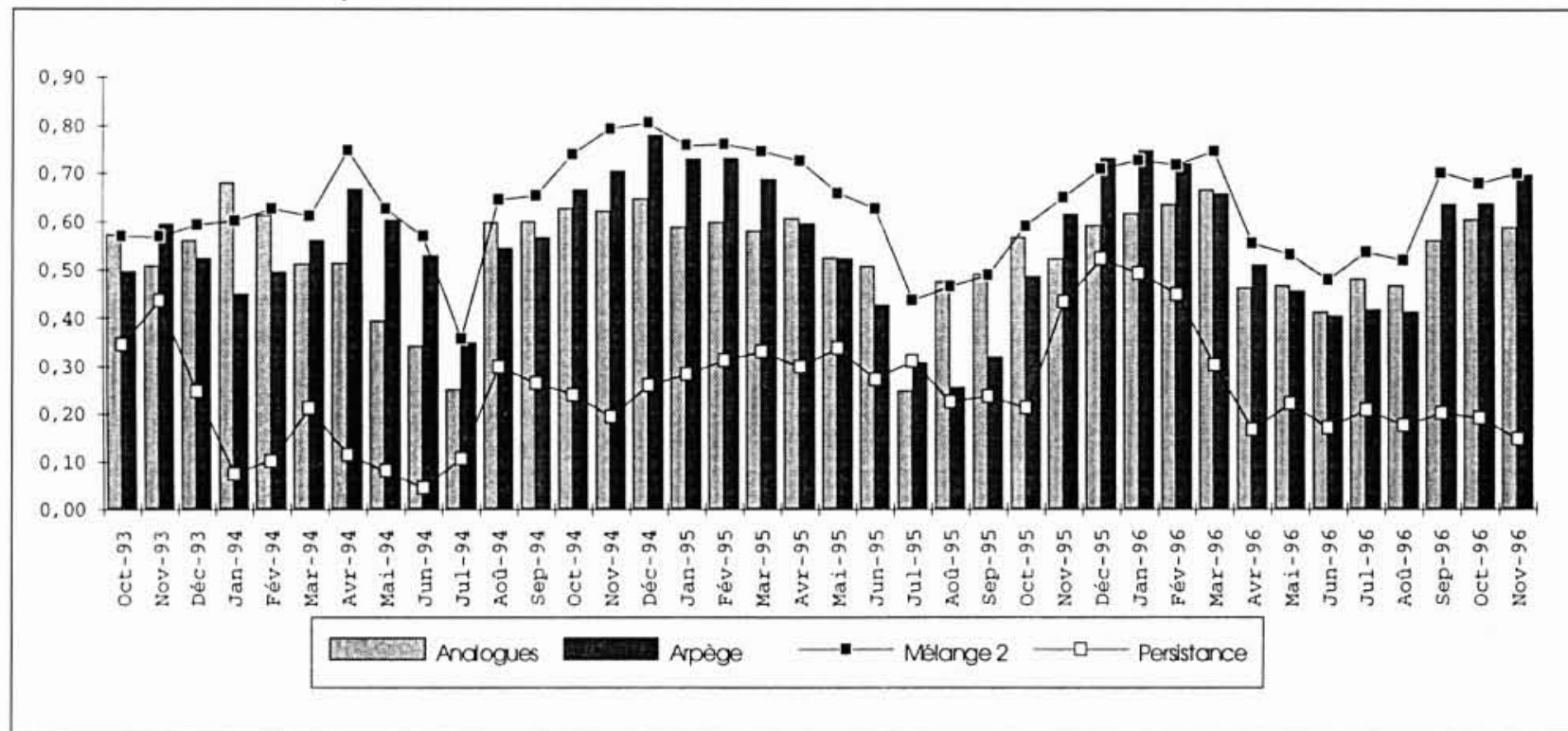

1. Etude temporelle des corrélations sur les prévisions alpines de précipitations en $\mathbf{2 4}$ heures (périodes de trois mois glissantes). 


\section{LE SYSTÈME MIS EN PLACE.}

L'ensemble de la fonction prévision repose donc sur Safran qui doit pouvoir fournir, sans donnée d'observation du jour à venir, les paramètres météo nécessaires à Crocus et Mépra. Pratiquement cela revient chaque matin à analyser la journée de la veille [6 UTC veille - 6 UTC matin] avec des observations disponibles sur cet intervalle passé et à enchaîner en mode prévision jusqu'à 6 UTC le lendemain. La solution mise en place repose en fait sur un panachage des deux méthodes du paragraphe précédent qui ont déjà été décrites [8] :

- utilisation du modèle Arpège après adaptation à notre échelle de travail via les opérateurs du paragraphe 1 qui servent habituellement à construire l'ébauche dans le cas d'analyse. D'autres opérateurs, adaptés aux précipitations, ont également été développés, car contrairement au cas d'analyse, les précipitations du modèle vont être utilisées.

- une recherche de situations analogues commençant l'été 1981. A partir des prévisions Arpège pour la journée nous cherchons l'ensemble $(=10)$ des situations analogues par comparaison avec des champs d'altitude préalablement stockés. La date de début de 81 repose justement sur la disponibilité numérique de ces archives au CEPMMT (date de début de fonctionnement) bien que beaucoup d'observations antérieures soient disponibles et que nous soyons convaincus que cette profondeur temporelle est encore trop courte du point de vue de la périodicité de retour de certains phénomènes. Les champs utilisés sont au nombre de 4 : géopotentiels $500 \mathrm{hPa}$ et $700 \mathrm{hPa}$, température et advection thermique (proche de la tendance temporelle) à $700 \mathrm{hPa}$. Différents opérateurs visant à fabriquer une distance sur les différences entre Arpège et les archives sont ensuite appliqués sur ces quatre champs ainsi que sur leurs dérivées (discrétisées) premières et secondes. Le domaine géographique de ces opérations de minimisation est malheureusement limité (Alpes et Pyrénées) par rapport aux références citées, ce qui peut gêner la reconnaissance de certaines structures synoptiques ; aussi la distance totale est-elle intégrée sur une durée de 30 heures sur le même (petit) domaine horizontal. Un terme supplémentaire fait intervenir (faiblement) les précipitations prévues et celles des archives. Le résultat final est donc une note dont les plus faibles valeurs permettent d'identifier la meilleure date d'analogues; il faut en effet se rappeler que cette note (ou distance) caractérise à la fois l'écart entre deux situations météo mais aussi la qualité de la prévision du modèle Arpège car on compare un ensemble de prévisions de 0 à $30 \mathrm{~h}$ d'échéance à des analyses du CEPMMT qui sont de meilleure qualité.

Après différentes expérimentations, on a trouvé que l'utilisation unique des prévisions d'Arpège adaptées à notre échelle était la meilleure solution pour l'estimation de tous les champs à l'exception des précipitations. Ce résultat a été longuement présenté dans [8] et reste d'actualité sur les Alpes et les Pyrénées. Nous pouvons l'illustrer brièvement en prenant l'exemple des températures au col de Porte (Chartreuse, $1340 \mathrm{~m}$ ) comme indiqué sur les figures 2 . La figure $2 A$ présente en tant que référence la restitution par la chaîne analyse de la température en ce point (sans en utiliser les observations) sur l'année 93-94 avec un score en $\mathrm{R}^{2}$ (carré de la corrélation) de 0,959 . La figure $2 B$ présente pour le même lieu aux mêmes dates le score de prévision à un jour par la méthode des analogues, le score en $R^{2}$ est alors de 0,764 . La figure $2 C$ illustre les performances d'Arpège également à un jour d'échéance qui atteint un $R^{2}$ de 0,895 .

La suite de ce papier va donc présenter le traitement particulier de la prévision de précipitations.

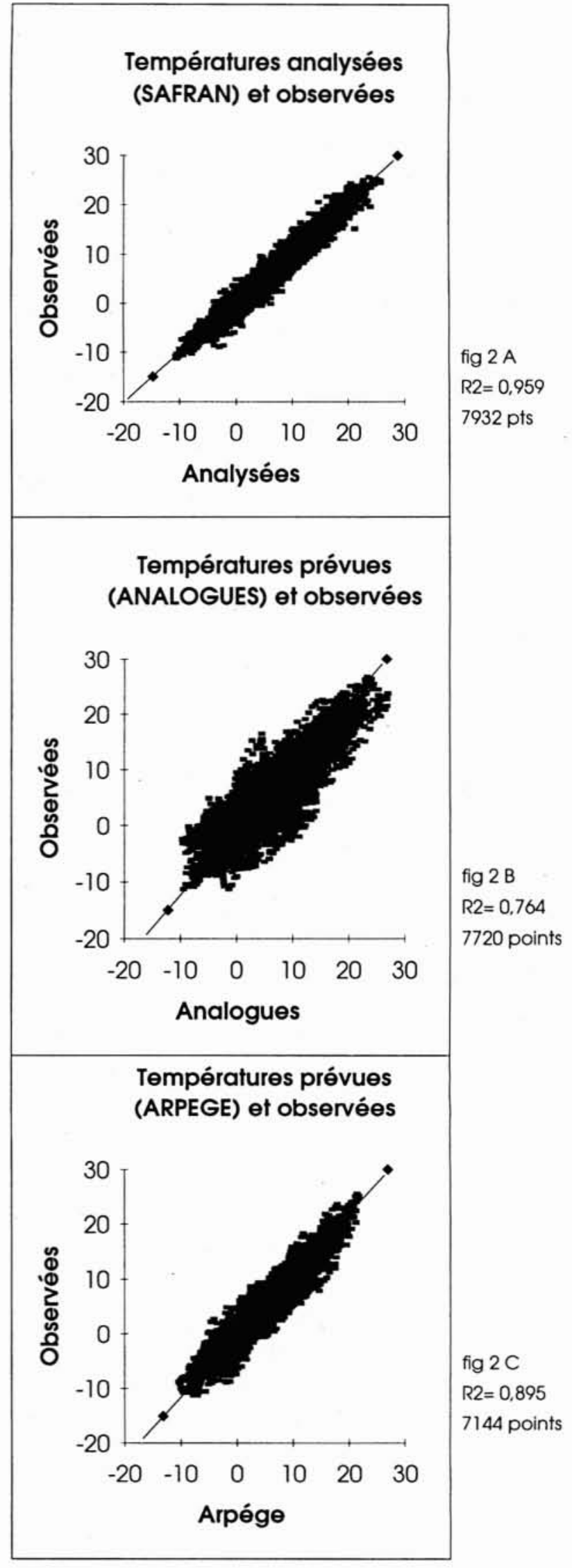

2. Col de Porte août 93 - Juillet 94 . 


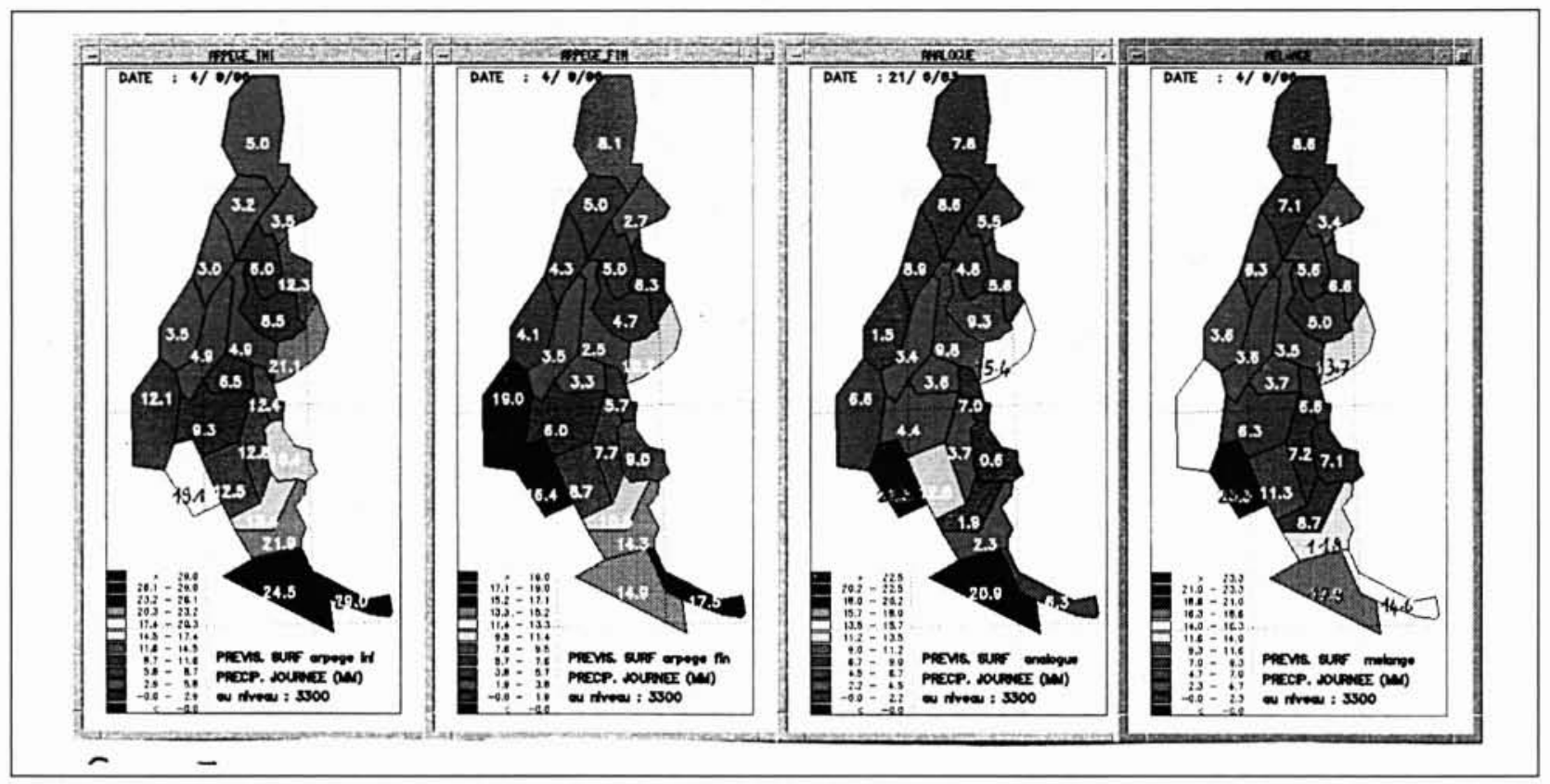

3. Visualisation des sorties en temps réel de la chaîne SCM.

\section{ILA PRÉVISION A UN JOUR DES CUMULS DE PRÉCIPITATIONS SUR $24 \mathrm{H}$}

Contrairement aux résultats du paragraphe précédent, nous avons vérifié que la méthode par analogue possédait une informativité qu'il fallait prendre en compte. Ce résultat semble compatible avec l'idée que l'apport des observations de la situation analogue permet de moduler selon les massifs la valeur «lissée » d'Arpège. La prévision Arpège a quant à elle l'avantage implicite d'être cohérente avec l'ensemble du système de prévision de MF et de ne pas présenter de déphasage temporel comme peut le faire notre application de la méthode des analogues qui recherche pour notre journée en cours [6 UTC-6 UTC] une autre journée sur le même intervalle temporel [6 UTC-6 UTC] alors que la meilleure solution est peut-être encore une autre journée sur un autre intervalle, par exemple [12 UTC-12 UTC] mais pour lequel nous n'avons pas d'observations de cumul de précipitations en $24 \mathrm{~h}$. Nous avons déduit des études présentées dans les prochains paragraphes que la solution résidait dans un mélange des deux prévisions possibles en essayant de garder leurs qualités respectives et de donner un résultat qui soit systématiquement meilleur.

\section{V ÉTUDES SUR LA QUALITÉ DES PRÉVISIONS DE PRÉCIPITATIONS D'ARPÈGE ET DES ANALOGUES}

Nous avons commencé par étudier la qualité intrinsèque des deux systèmes de prévisions à la fois sur les Alpes avec un fichier d'apprentissage de près de trois ans ( 816 couples $)$ et sur les Pyrénées avec une profondeur d'un peu plus d'un an ( 326 couples). Cette vérification s'effectue sur l'ensemble des massifs de la PRA (23 dans les Alpes, 15 sur les Pyrénées, cf. fig. 4) et prend comme référence l'analyse Safran effectuée le jour suivant lorsque les observations sont disponibles. Ce choix est discutable mais commode; la variabilité naturelle du champ de précipitation en montagne fait qu'il est difficile de comparer directement nos prévisions à des observations et l'utilisation d'une analyse comme référence permet d'effectuer des comparaisons à une échelle identique (le massif) différente de l'échelle locale des observations et à une même altitude $(1800 \mathrm{~m})$. Ce système de comparaisons peut cependant aussi être justifié par les résultats des études entre précipitations observées et analysées au Col de Porte et présentées dans [8].

Les premières comparaisons concernant les valeurs moyennes montrent sur nos deux domaines une énorme similitude entre analyse de référence et prévision par analogues comme illustré par la figure 4. Nous sommes là sur un artefact de notre système de comparaison ; nous vérifions que les deux analyses (celle en temps réel de vérification et celle sur la situation analogue passée) possèdent bien la même climatologie interne (selon 7 types de temps sur les Alpes et 9 sur les Pyrénées). La valeur prévue par Arpège est elle bien différente ; elle est plus importante en moyenne et est fortement influencée par le relief propre du modèle (cf. fig. 4). Ainsi les massifs à altitude élevée dans le modèle (par exemple Haute Maurienne dans les Alpes ou les massifs espagnols dans les Pyrénées) sont systématiquement surévalués dans l'estimation d'Arpège qui donne une indication représentative des précipitations à une altitude plus élevée que celle de comparaison $(1800 \mathrm{~m})$. Cette première étude nous a cependant permis d'établir des coefficients de recalage (ou débiaisage) pour chaque massif de l'estimation d'Arpège avec une variabilité saisonnière sur les Alpes et annuelle sur les Pyrénées. Ils seront bien sûr révisés lorsque la profondeur des fichiers d'apprentissage sera meilleure.

La seconde étude a porté sur la véracité de la prévision du phénomène " pluie » pour la journée du lendemain, indépendamment de la quantité prévue. Cela revient à étudier une simple table de contingence à deux entrées caractérisant l'occurrence du phénomène. L'indice de vérification utilisé est celui de Rousseau [9] qui est bien adapté aux précipitations et varie entre 0 (très mauvais) et 1 (prévision parfaite). Contrairement au critère classique de calcul de la première diagonale, il est plus contraignant et pénalise systématiquement les prévisions biaisées; en plus sa formulation nonlinéaire est compatible avec la définition d'une distance entre plusieurs séries. Les résultats ont finalement montré que Arpège n'était le meilleur que sur les Pré-Alpes du Cha- 

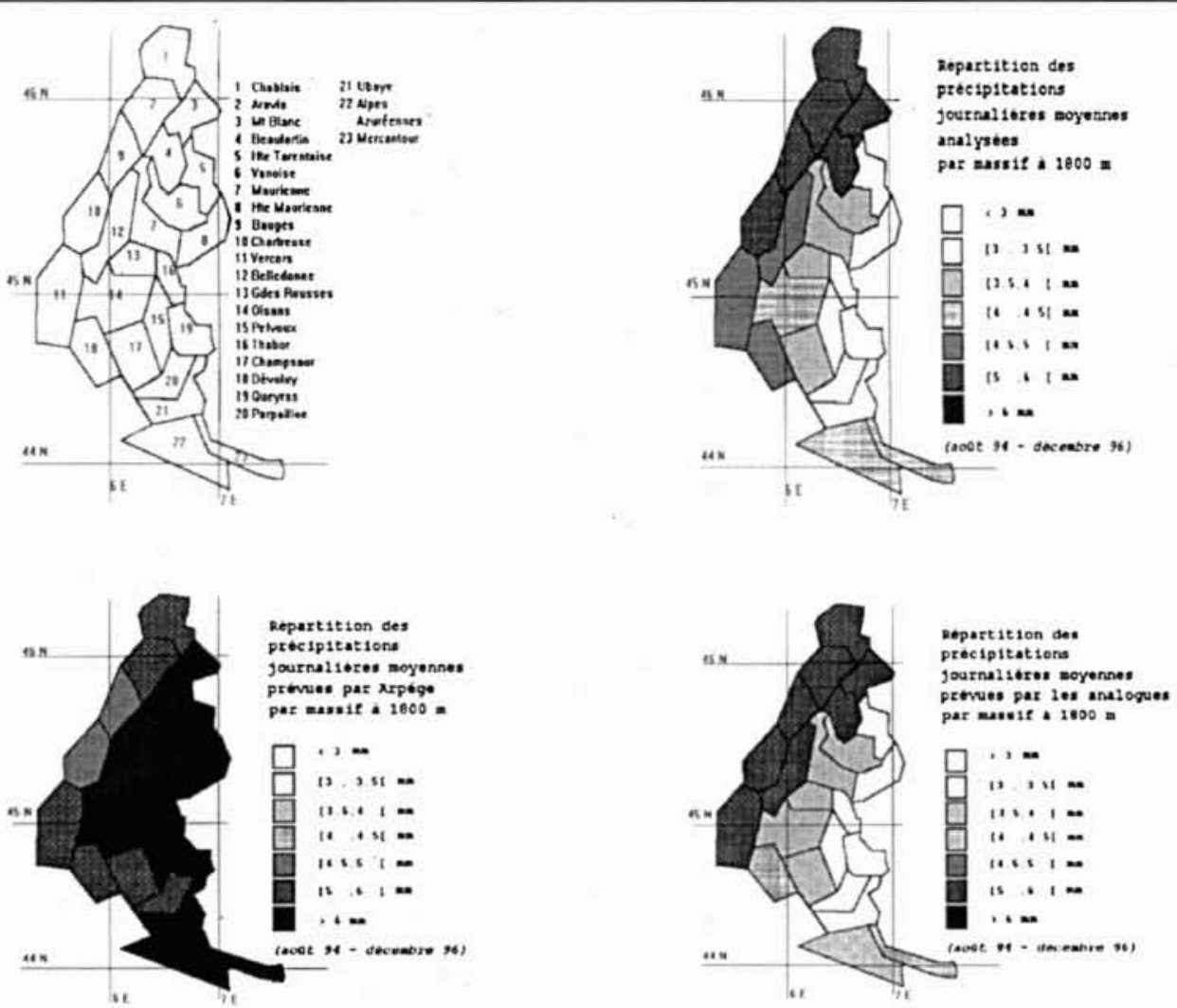

Domaine Alpes (23 massifs). précipitations moyennes journalières (en haut).

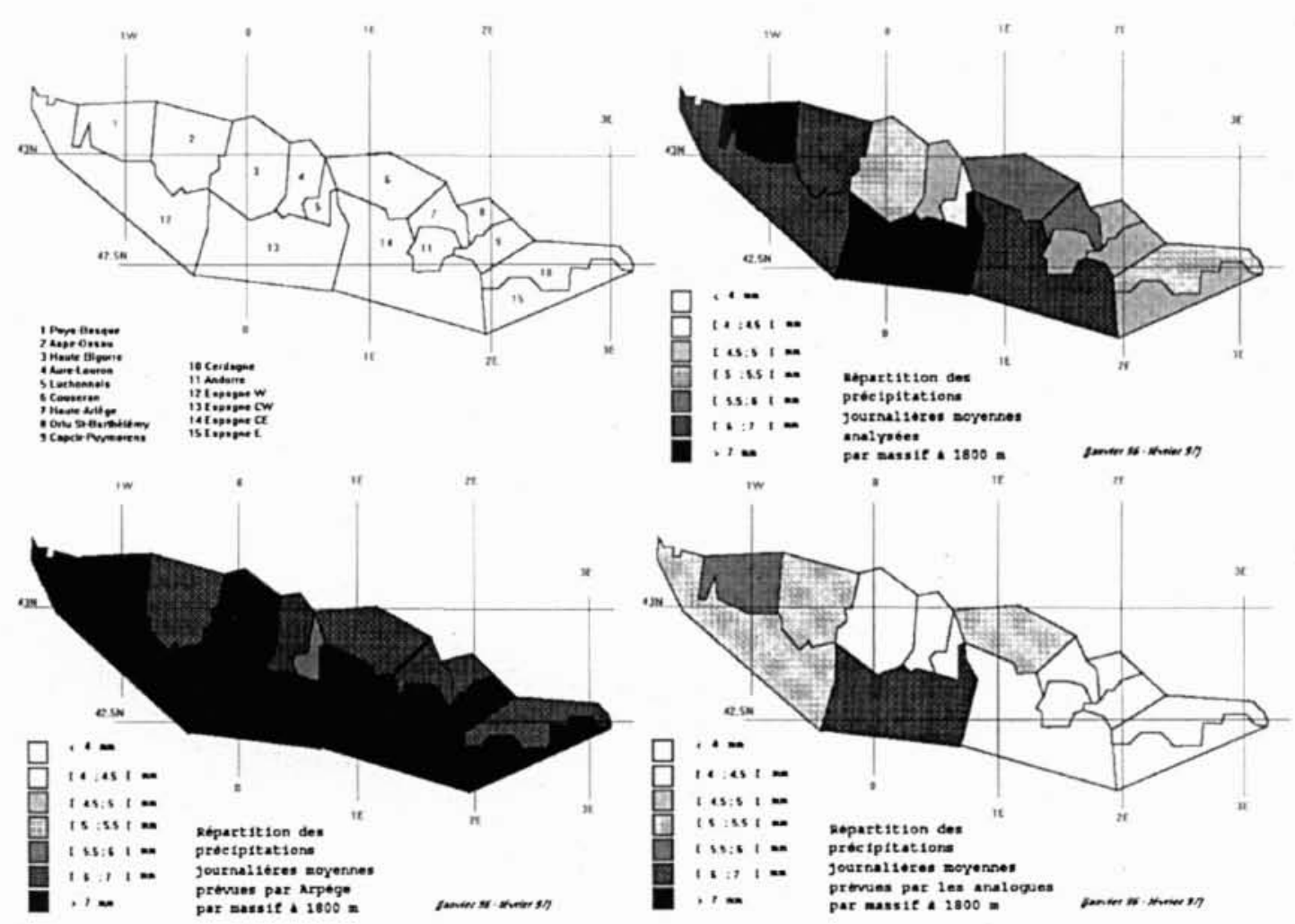

Domaine Pyrénées (15 massifs). Précipitations moyennes journalières (en bas).

4. Répartition moyenne des précipitations journalières. 
blais à la Chartreuse et sur la partie occidentale de la chaîne pyrénéenne (Pays-Basque, Aspe-Ossau). Ce résultat semble en accord avec le trajet "classique " des perturbations abordant ces massifs et la bonne représentation de ces perturbations par le modèle. Cependant le modèle semble après gérer plus mal la progression (ou le blocage) des zones précipitantes à l'intérieur des massifs, tandis que les analogues y sont sensiblement meilleurs.

Une troisième étude sur une table de contingence à 5 classes de précipitations $([0]] 0,1]] 1,5]] 5,10,],\{>10\})$ a globalement confirmé l'impression précédente en montrant la supériorité de la répartition d'Arpège sur les premiers massifs frontaliers ouest à la fois dans les Alpes et les Pyrénées et celle des analogues dans les autres massifs.

Nous avons ensuite étudié les corrélations entre champs prévus et analysés par massifs. Cette étude est plus difficile à interpréter en valeur absolue car notre champ de référence (l'analyse) est forcément légèrement erroné en raison de sa propre technique d'interpolation ou par l'utilisation de données fausses ou plus prosaïquement par le manque d'observation en dehors de la saison nivologique. Mais, comme mentionné précédemment, le principal avantage est que nous n'avons pas trop de problème d'échelle. A l'opposé des vérifications précédentes, cette étude est nettement en faveur d'Arpège qui montre un comportement supérieur sur la grande majorité des massifs Alpins et Pyrénéens. Les principales faiblesses d'Arpège apparaissent dans les massifs situés du côté Est des Alpes comme la Haute Tarentaise. Dans ces massifs en effet, le modèle apprécie mal les situations de sud et de sud-ouest pour lesquelles il propose de fortes précipitations alors que dans la réalité elles sont faibles ou peu significatives avec un régime de foehn plus ou moins marqué sur la frontière (communication personnelle des prévisionnistes de Bourg St Maurice). Dans les Pyrénées, seuls l'Andorre et un massif central espagnol sont mieux vus par les analogues que par Arpège.

Une brève synthèse des résultats est montrée dans le tableau 1 ; elle illustre les caractéristiques moyennes des adaptations Arpège (notées $\mathrm{P}$ ) et celles des analogues $(\mathrm{S})$ par comparaison avec les analyses (A). Il faut cependant noter que ces paramètres peuvent présenter de fortes variations annuelles à l'instar des corrélations moyennes sur l'ensemble des Alpes montrées en figure 1 par trimestre glissant. Sur ce dernier graphique, quoique limité à 3 ans, on apprécie bien les variations de performance annuelle. Les relatifs mauvais scores estivals sont principalement dus au caractère convectif des précipitations que les deux méthodes ont du mal à appréhender, tandis que la corrélation dépasse la plupart du temps 0,75 en période hivernale. L'apport de chaque méthode est apprécié relativement à la méthode frustre de prévision par persistance.

\section{VI — LE MÉLANGE}

La synthèse des évaluations du paragraphe précédent ainsi que d'autres (non présentées) réalisées sur des périodes plus courtes mais correspondant à des saisons à régimes pluviométriques différents (convectif, frontal, mixte) nous a guidé dans notre méthode de panachage des deux solutions. Le principe retenu a été de minimiser la variance de la différence entre l'analyse et une combinaison linéaire des deux solutions préalablement centrées; une conséquence immédiate a été l'obtention automatique d'une corrélation supérieure à celle de chacune des deux solutions prises isolement. Ce mélange optimal prenant de l'information dans chacune des deux solutions, produit une combinaison de meilleure qualité que chacun des composants (tabl. l, paramètre M1). Les coefficients de la combinaison linéaire sont variables selon les massifs et sont annuels sur les Pyrénées et saisonniers sur les Alpes.

$$
R f 1=\alpha N a R a+(1-\alpha) N p R p
$$

avec : $R f 1=$ Résultat final, $R a=$ Prévision par analogues, $R p=$ Prévision Arpège

et $\alpha=$ Coefficient de combinaison

et $\mathrm{Na}=$ facteur de normalisation des analogues, $\mathrm{Np}=$ Facteur de normalisation d'Arpège.

Les coefficients de combinaisons montrent que dans chaque massif le mélange est à l'avantage d'Arpège qui a un poids plus important que les analogues. Ce fait est encore plus marqué dans les Pyrénées que dans les Alpes. On peut donc interpréter ce mélange comme le résultat d'un champ de grande échelle renormalisé par massif et en phase avec la réalité météorologique (Arpège) pondéré par des analogues moins bons intrinsèquement mais capables d'apporter une information de plus petite échelle à la fois sur l'occurrence du phénomène et sur la variabilité entre les massifs.

\section{L'ALGORITHME DANS LES CAS DE PRÉVISIONS DIVERGENTES}

Le résultat obtenu Rf1 est donc totalement « performant » du point de vue du critère de la corrélation avec nos analyses et l'utilisation de coefficients saisonniers nous permet d'être sûr d'avoir une certaine qualité en hiver lorsque l'analyse de référence est fiable. A l'opposé, ce mélange présente des caractéristiques de contingence (cf. § précédent) totalement déplorable dans les cas étudiés à 2 et 5 classes. L'explication est simple: nous avons cumulé les fausses prévisions de

Tableau 1. - Vérification des analyses/analogues/Arpège en RR journaliers.

\begin{tabular}{|c|c|c|c|c|c|c|c|c|c|c|c|c|c|}
\hline & $\begin{array}{l}\mathrm{M} \\
\mathrm{A}\end{array}$ & $\begin{array}{c}M \\
S\end{array}$ & $\begin{array}{l}M \\
P\end{array}$ & $\begin{array}{l}\mathrm{M} \\
\mathrm{MI}\end{array}$ & $\begin{array}{l}\mathrm{M} \\
\mathrm{M} 2 \\
\end{array}$ & $\begin{array}{c}C O \\
(A, S)\end{array}$ & $\begin{array}{c}C O \\
(A, P)\end{array}$ & $\begin{array}{c}\mathrm{CO} \\
(\mathrm{A}, \mathrm{MI})\end{array}$ & $\begin{array}{c}\mathrm{CO} \\
(\mathrm{A}, \mathrm{M} 2)\end{array}$ & $\begin{array}{c}\text { Ro2 } \\
(A, S)\end{array}$ & $\begin{array}{r}R \circ 2 \\
(A, P)\end{array}$ & $\begin{array}{c}\text { Ro2 } \\
(\mathrm{A}, \mathrm{Ml})\end{array}$ & $\begin{array}{c}\mathrm{Ro2} \\
(\mathrm{A}, \mathrm{M} 2)\end{array}$ \\
\hline TOUT_ALP & 4,20 & 4,13 & 7.23 & 4,54 & 4,46 & 0,55 & 0,56 & 0,67 & 0.67 & 0,48 & 0,37 & 0,30 & 0,53 \\
\hline ANDORRE & 4,55 & 2.92 & 7.27 & 4,53 & 3.90 & 0,50 & 0,46 & 0,58 & 0,57 & 0.29 & 0,24 & 0,13 & 0,34 \\
\hline TOUT_PYR. & 5.67 & 4,48 & 7,47 & 5,67 & 5.67 & 0,36 & 0,52 & 0.57 & 0,58 & 0.37 & 0,32 & 0,22 & 0,49 \\
\hline FRANCE-PYR & 5.34 & 4,49 & 7.06 & 5.36 & 5.40 & 0.35 & 0,56 & 0.59 & 0,60 & 0,40 & 0,35 & 0,25 & 0,52 \\
\hline ESPAGNE-PY & 6,75 & 4,84 & 8.53 & 6,74 & 6,77 & 0,34 & 0,47 & 0,54 & 0,53 & 0,33 & 0.28 & 0,17 & 0,44 \\
\hline
\end{tabular}

M=Moyenne Journaliére $\quad \mathrm{CO}=$ Corrélation $\quad$ Ro2=Critère Rousseau 2 classes

$A=$ Analyse (Safran) de Référence

S=Prévision (Safran) par Analogues

$\mathrm{Ml}=$ Mélange Prévision SANS algorithme

$P=$ Prévision Arpége
M2=Mélange Prévision AVEC algorithme

Alpes : 
chacun des deux composants du mélange. Ainsi il suffit que l'un des deux fasse une fausse prévision de précipitations pour le lendemain pour que le mélange soit également pluvieux et faux. En corollaire pour réussir une prévision de «non-pluie » il faut que les deux composants soient également « secs ». Tout cela se traduit pratiquement par une diminution drastique du critère de Rousseau appliqué à l'évaluation des tables de contingence du mélange qui illustre pratiquement que la variable bornée "précipitation » est représentative d'une discontinuité " physique " quand sa valeur est à zéro ou non.

L'amélioration de ce premier résultat intermédiaire est donc indispensable et a été réalisée par un algorithme qui vise à résoudre le cas où pour un massif une méthode donne un diagnostic de pluie et l'autre de temps sec. La procédure de correction est basée sur le critère de Rousseau qui nous a indiqué $(\$ 5)$ les massifs respectifs où chacune des méthodes appréciait au mieux l'occurrence de précipitation. Le principe est alors de prendre uniquement (sans mélange) dans chaque massif la méthode la plus appropriée (selon Rousseau) quand les deux estimations different dans leurs prévisions d'occurrence de précipitations. La qualité "moyenne" meilleure d'Arpège apparaît cependant dans l'algorithme : on garde en effet une prévision de fortes précipitations d'Arpège même dans le cas de massifs où l'on aurait dó prendre une prévision "sèche" des analogues selon le critère précédent. L'ensemble de ces règles est appliqué dans les Alpes et les Pyrénées et conduit à la fabrication d'un champ Rf2 qui se révèle supérieur aux autres estimations pour tous les paramètres statistiques précédemment évoqués (moyenne, corrélation, critère de Rousseau) comme présenté dans le tableau 1 (paramètre M2) et sur la figure 2.

\section{VIII — L'UTILISATION ROUTINIÈRE}

La visualisation graphique des sorties en temps réel de la chaîne SCM n'est effective qu'à la station de Grenoble. Les autres centres en disposerons bientôt ; ils reçoivent actuellement un ensemble de messages précisant les quantités de précipitations prévues dans leurs massifs et les risques Mépra pour la journée du lendemain. La future représentation graphique présente quant à elle un ensemble complet à la fois sur les variables météo, nivo et les risques prévus d'avalanche. Une des images illustre les deux composantes du mélange de prévision de précipitations. Elle est présentée sur la figure 3, malheureusement sans les couleurs habituelles. On y trouve de gauche à droite la version initiale de l'adaptation d'Arpège, puis le même champ après interpolation verticale et débiaisage. Le troisième champ est la prévision par analogues avec une indication de la date sélectionnée. Le champ de droite est le mélange après correction algorithmique.

\section{CONCLUSION}

Nous avons montré une «n-ième " fois que la prévision en montagne est un art difficile dans lequel nous avons encore beaucoup à apprendre. Notre tentative, bien que basée sur des séries temporelles bien trop courtes, répond parfaitement aux impératifs d'une prévision locale à échelle plus fine que celle des modèles numériques globaux comme Arpège.
L'apport de la prévision analogue est à la fois «sensible " car le prévisionniste apprécie de pouvoir se rattacher à une situation du passé qui est un des éléments de sa propre expertise et il est aussi « discriminant " pour l'occurrence du phénomène précipitation.

\section{REMERCIEMENTS}

Un système aussi complet que la chaîne SCM ne serait qu'un ensemble vide s'il n'était pas alimenté en temps réel par les observateurs du réseau nivo-météo qui fournissent les indispensables observations sur les Alpes et les Pyrénées et que nous essayons ensuite d'extrapoler pour la journée du lendemain... avec plus ou moins de réussite.

\section{RÉFÉRENCES}

[1] Brun E., Martin E., Simon V., Gendre C., Coléou C. (1989). - An energy and mass model of snowcover suitable for operational avalanche forecasting. J. OF GLACIOL., VoL. 35 , $N^{*} 121$, P. $333-342$.

[2] Brun E., David P., Sudul M., Brunot G. (1992). - A numerical model to simulate snowcover stratigraphy for operational avalanche forecasting, J. of Glaciol., Vol. 38, $\mathrm{N}^{*} 128$, p. 13-22.

[3] GIRAUD G. (1992.) - MEPRA : An expert system for avalanche risk forecasting. Proceedings of the International snow science workshop, 4-8 oct. 1992, Breckenridge, Colorado, USA, p. 97-106.

[4] Durand Y., Brun E., Mérindol L., Guyomarc'h G., LeSAFFre B., MARTIN E. (1993), - A meteorological estimation of relevant parameters for snow models, Annals of Glaciol., Vol. 18 , p. $65-71$.

[5] Courtier P., Freydier C., Geleyn J-F., Rabier F, et Rochas M. (1991). - The Arpege project at Météo-France. ECMWF Seminar Proceedings 9-13 septembre 1991, Vol. 2, pp. 193-232.

[6] Duband D. (1981). - Prévision spatiale des hauteurs de précipitations journalières. La Houille Blanche, juillet/août 1981, pp. 497-511.

[7] NaVARre J.P. (1980). - Prévision des précipitations sur les Alpes par une analyse des situations analogues. Proceeding du $16^{\mathrm{e}}$ congrés international de Météorologie Alpine. Aix-les Bains, sept. 1980, pp. 209-213.

[8] Durand Y., Mérindol L., Michoud S. (1995), - Premiers éléments pour une prévision du risque d'avalanche au cours de la journée du lendemain. Proceedings du Symposium International sur les apports de la recherche scientifique à la sécurité neige, glace et avalanches. Chamonix 30/5-3/6/95 p. 169-176. Disponible auprès de l'ANENA (Grenoble).

[9] Rousseau D. (1985). - Compléments sur un critère de vérification de prévisions catégoriques. Note de Travail n ${ }^{*} 123$ de l'Etablissement d'Etudes et de recherches Météorologiques, Météo-France. 